\title{
Dynamic optically induced planar terahertz quasioptics
}

\author{
Cooke, David; Jepsen, Peter Uhd
}

Published in:

Applied Physics Letters

Link to article, DOI:

$10.1063 / 1.3153988$

Publication date:

2009

Document Version

Publisher's PDF, also known as Version of record

Link back to DTU Orbit

Citation (APA):

Cooke, D., \& Jepsen, P. U. (2009). Dynamic optically induced planar terahertz quasioptics. Applied Physics Letters, 94(24), 241118. https://doi.org/10.1063/1.3153988

\section{General rights}

Copyright and moral rights for the publications made accessible in the public portal are retained by the authors and/or other copyright owners and it is a condition of accessing publications that users recognise and abide by the legal requirements associated with these rights.

- Users may download and print one copy of any publication from the public portal for the purpose of private study or research.

- You may not further distribute the material or use it for any profit-making activity or commercial gain

- You may freely distribute the URL identifying the publication in the public portal

If you believe that this document breaches copyright please contact us providing details, and we will remove access to the work immediately and investigate your claim 


\title{
Dynamic optically induced planar terahertz quasioptics
}

\author{
D. G. Cooke ${ }^{\text {a) }}$ and P. Und Jepsen \\ DTU Fotonik, Technical University of Denmark, Building 343 Ørsted Plads, 2800 Kgs. Lyngby, Denmark
}

(Received 29 April 2009; accepted 22 May 2009; published online 19 June 2009)

\begin{abstract}
Optical control of the propagation direction of a terahertz pulse inside an optically transparent parallel plate waveguide is demonstrated by patterned charge carrier photoexcitation of a silicon slab embedded within the waveguide. It is shown experimentally and through finite element simulations that photoexcitations with sufficient conductivity can induce a partial reflection, capable of steering the pulse inside the two-dimensional waveguide. A beamsplitter is demonstrated as proof of principle and is used to delay the arrival of the reflected terahertz pulse at the detector by several picoseconds by moving the excitation in the plane. () 2009 American Institute of Physics. [DOI: $10.1063 / 1.3153988]$
\end{abstract}

The terahertz (THz) region, lying between $300 \mathrm{GHz}$ and $10 \mathrm{THz}$, is the last remaining commercially unexploited part of the electromagnetic spectrum. High speed communications and lab-on-a-chip sensing have been noted among the many applications making use of $\mathrm{THz}$ radiation. ${ }^{1}$ However, the lack of basic components for this region of the spectrum, such as modulators and switches, hinders the progress of these promising technologies. Several research groups are dedicated to filling in this gap, and there has been significant progress particularly in the field of THz metamaterials ${ }^{2-4}$ and waveguides. ${ }^{5-10}$

The parallel plate waveguide (PPWG) has shown itself to be a nearly ideal two-dimensional (2D) interconnect for low loss, zero dispersion pulse propagation. ${ }^{6}$ By incorporating $2 \mathrm{D}$ quasioptics between the plates, pulses can be manipulated in the plane just as in free space as the transverse electromagnetic (TEM) mode has no spatial dependence perpendicular to the plates. ${ }^{11}$ Reflective, ${ }^{11}$ refractive, and diffractive quasioptical elements ${ }^{12}$ have been demonstrated as well as 2D photonic crystal lattices, ${ }^{13,14}$ waveguides, ${ }^{9}$ and resonators. ${ }^{15}$ These passive components form the basic structure of a $\mathrm{THz}$ photonic circuit, all on a chip. A key missing feature, however, is the ability to actively control the direction of pulse propagation in the plane. Recently, we reported on optical modulation of $\mathrm{THz}$ pulse transmission inside a PPWG. ${ }^{16}$ This was accomplished by replacing one or both of the opaque metallic plates with a transparent conducting oxide, which allowed laser excitation of an embedded semiconductor slab through the transparent plate. The photoinduced charge carriers then attenuate the $\mathrm{THz}$ pulse transmission through the PPWG by free-carrier absorption. We then showed how time-resolved spectroscopy of injected photocarriers can be performed within the waveguide, which can increase sensitivity and allow spectroscopy on small sample volumes. ${ }^{17}$

In this paper, we demonstrate how this technique can be used to not only attenuate $\mathrm{THz}$ transmission but to induce a reflective element capable of steering the pulse within the waveguide. Provided the excitation is pulsed and the $\mathrm{THz}$ pulse traverses the region of photoconductivity before charge carriers can diffuse, a conducting region that is sharp com-

\footnotetext{
a) Author to whom correspondence should be addressed. Electronic mail: dcoo@fotonik.dtu.dk.
}

pared to the wavelength of $\mathrm{THz}$ light can induce a reflection. This is very similar to the known reflection of ultralow frequency waves off the Gaussian-like conductivity profile in the Earth's ionosphere. ${ }^{18}$ We experimentally demonstrate pulse steering by effectively creating a beamsplitter inside the waveguide with a line focus photoexcitation of an embedded silicon slab. We show proof of principle through movement of the beamsplitter in the plane, causing a delay in the arrival of the reflected pulse to the detector by several ps. Finally, the concept is explored computationally by $2 \mathrm{D}$ finite element simulations, where the transmission, reflection, and absorption coefficients are calculated for a Gaussian line conductivity profile at $45^{\circ}$ to the incoming radiation.

A thin film PPWG is fabricated according to previous work, ${ }^{17}$ by coating a polished, $100 \mu \mathrm{m}$ thick, high resistivity silicon wafer $(\rho>10000 \Omega \mathrm{cm})$ on one side with $300 \mathrm{~nm}$ of Au with a $2 \mathrm{~nm}$ Ti adhesion layer, and the other side with $1.6 \mu \mathrm{m}$ of fluorinated tin oxide (FTO), a transparent conducting oxide. The static transmission properties have previously been reported, with power absorption coefficients of $4 \mathrm{~cm}^{-1}$ at $0.5 \mathrm{THz}$ and practically no dispersion across the THz bandwidth. ${ }^{17}$ Coupling into and out of the thin film waveguide is achieved through a second Al PPWG using the thin film PPWG as a spacer, just as in Ref. 17 and shown in Fig. 1(a) without the front $\mathrm{Al}$ plate for clarity. A 5 $\times 5 \mathrm{~mm}^{2}$ square hole is cut in the front Al plate to allow optical access to the FTO side of the thin film PPWG, which bridges the hole. The waveguide assembly sits in the focal region of a typical $\mathrm{THz}$ time-domain spectrometer, based on optical rectification for $\mathrm{THz}$ generation in a $1 \mathrm{~mm}$ thick $\mathrm{ZnTe}$ crystal and detected by free-space electro-optic detection in a second $0.5 \mathrm{~mm}$ thick ZnTe crystal.

The 45 fs duration, $1 \mathrm{kHz}$ repetition rate, output from an amplified fs Ti:sapphire laser system is split into three beams, one used as a source beam for $\mathrm{THz}$ pulses, and a second as a gating beam for $\mathrm{THz}$ detection. A third beam is used to pump an optical parametric amplifier to frequency convert the $800 \mathrm{~nm}$ fundamental to $1030 \mathrm{~nm}$ by second harmonic generation of the idler. The reason for this frequency conversion is to provide a uniform excitation profile through the thickness of the slab, as the optical penetration depth for $1030 \mathrm{~nm}$ wavelength light in silicon is approximately $100 \mu \mathrm{m}$. The average power of the pump beam was $2.5 \mathrm{~mW}$ before the FTO coating, which transmits approximately $20 \%$ 


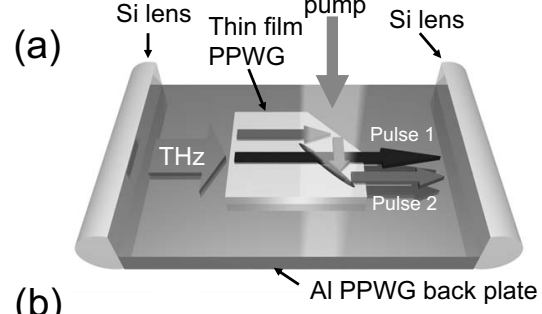

(b)

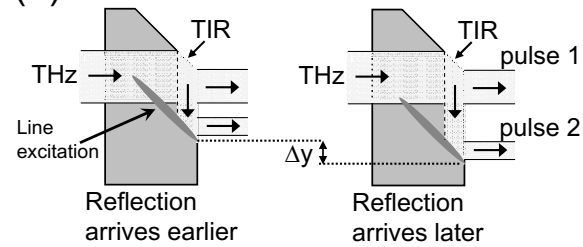

FIG. 1. (a) Schematic of the diced thin film PPWG inside the Al PPWG (top plate removed for clarity) showing a line focus pump beam acting as a beam splitter. (b) Schematic showing the geometry of the $\mathrm{THz}$ pulse propagation inside the thin film PPWG, the splitting of the input pulse into two pulses by TIR and the delay of the reflected portion of the pulse with respect to a transmitted pulse.

of the $1030 \mathrm{~nm}$ pump fluence. The pump beam is focused to a $2 \mathrm{~mm}$ long $\left(1 / e^{2}\right)$ line by a $30 \mathrm{~cm}$ cylindrical lens with focal position located at the thin film PPWG. The beam waist of the resulting line is approximately $32 \mu \mathrm{m}$, calculated by Gaussian beam optics. The cylindrical lens is oriented such that the line excitation is $45^{\circ}$ to the incoming $\mathrm{THz}$ pulse, and parallel to a diced edge in the thin film PPWG, shown in Fig. 1 , which was subsequently hand polished with $0.5 \mu \mathrm{m}$ grit polishing paper. Care was taken in the polishing to achieve a perpendicular edge, as any wedge can lead to coupling to lossy, higher order modes. ${ }^{11}$ The THz beam is partially incident on the diced slab edge, such that the lower part of the beam is transmitted straight to the detector (pulse 1) and the upper part of the beam is reflected downwards in the slab (pulse 2) by total internal reflection (TIR), as shown in Fig. 1(b). If the pump pulse is present, the created charge carrier profile can induce a reflection that redirects the downward traveling pulse 2 toward the detector, which appears delayed by several ps with respect to the pulse 1 . The line focus is vertically displaced by a distance $\Delta y$ [see Fig. 1(b)] in the plane by rotation of the vertical adjustment of a mirror following the cylindrical lens in steps of $1 / 8$ of a rotation, and then calculating the displacement by the 80 turns per inch motion of the screw, knowing the distance of the screw to the pivot point of the mirror. The delay of pulse 2 is then expected to increase linearly with $\Delta y$ if it is caused by a reflection from the pump line excitation within the waveguide, due to the longer path length taken inside the slab to the detector, as depicted in Fig. 1(b). As pulse 1 is transmitted through the line focus directly to the detector, its arrival time is unaffected by the motion of the line focus.

Figure 2(a) shows the experimental results, mechanically chopping the optical pump beam so that only the pumpinduced modulation, $\Delta E=E_{\text {unpumped }}-E_{\text {pumped }}$, of the detected $\mathrm{THz}$ pulses are measured. Two modulated pulses are observed, an earlier pulse whose arrival time at the detector is unaffected by the vertical displacement of the pump beam, and a second pulse which is increasingly delayed with increasing $\Delta y$. The earlier pulse is simply the THz pulse transmitted directly to the detector, modulated by transmission through the line focus (pulse 1 in Fig. 1). The two pulses are in phase with respect to one another, as no phase shift is
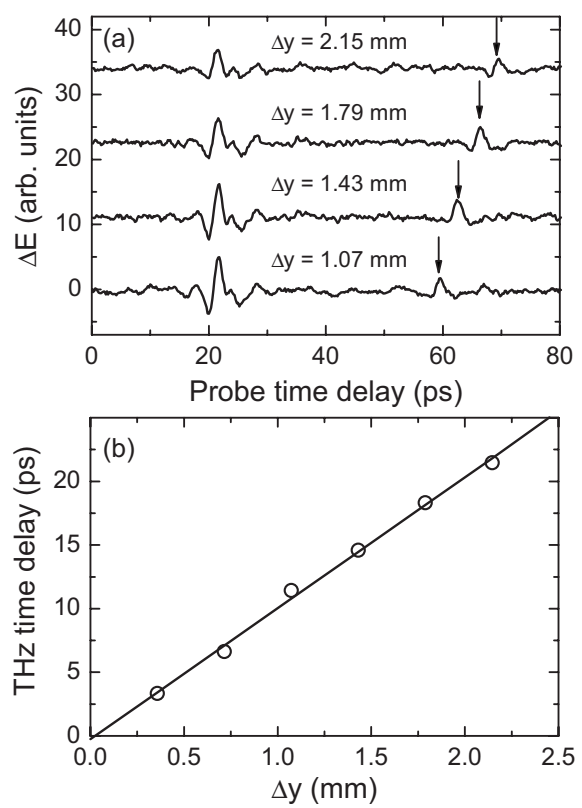

FIG. 2. (a) Detected pump-induced modulation $(\Delta E)$ of $\mathrm{THz}$ pulses for several vertical displacements of the pump beam line focus. (b) Corresponding $\mathrm{THz}$ peak positions as a function of vertical displacement, with linear fit.

expected for the TIR of light polarized perpendicular to the plane of reflection. ${ }^{19}$

The arrival time of pulse 2 is plotted in Fig. 2(b) and is linear with $\Delta y$, as is expected for the reflected geometry in Fig. 1. The expected time delay is given by $\Delta t=n_{\mathrm{Si}} \Delta y / c$, where $n_{\mathrm{Si}}$ is the index of refraction of $\mathrm{Si}$ at $\mathrm{THz}$ frequencies (3.42).$^{20} \mathrm{~A}$ linear fit of this equation to the data is shown in Fig. 2(b), yielding $n_{\mathrm{Si}}=3.05,10 \%$ lower than the expected 3.42. A possible source of this discrepancy is the walk-off induced by the reflection as the line focus is moved downwards, changing the timing of the detection to earlier times. Furthermore, the detection of pulse 2 was found to be extremely sensitive to the angle of the line focus with respect to the diced edge. Combined with the linear time shift of the second pulse with $\Delta y$, this is a clear signature of a pumpinduced reflection changing the propagation direction of the $\mathrm{THz}$ pulse within the slab, and is the first demonstration of optically controllable $\mathrm{THz}$ beam steering in a planar waveguide.

A 2D finite element simulation using the harmonic solver of the commercially available COMSOL software package is shown in Fig. 3, for a $0.5 \mathrm{THz} \mathrm{TM}$ polarized plane wave incident on a line of conducting region in silicon, defined as a Gaussian profile $\sigma(r)=\sigma_{0} \exp \left[-(r / w)^{2}\right]$, where $\sigma_{0}$ is the peak dc conductivity and $r$ is the perpendicular coordinate from the line direction. Figure 3(a) shows the 2D conductivity plot used in the simulation, tilted by $45^{\circ}$ relative to the incoming wave. Nonuniform meshing was used to ensure that the skin depth of the excitation was sufficiently meshed. The Gaussian waist, $w$, was $32 \mu \mathrm{m}$ to match experimental conditions. The form of the complex conductivity, $\sigma=\sigma_{1}$ $+\mathrm{i} \sigma_{2}$, is assumed to be a Drude form, and both $\sigma_{1}$ and the dielectric function, $\varepsilon_{1}=\varepsilon_{B}-\sigma_{2} / \varepsilon_{0} \omega$, are taken into account with a scattering time of $50 \mathrm{fs}$, typical of photoexcited $\mathrm{Si}$ at room temperature. The background dielectric constant, $\varepsilon_{\mathrm{B}}$, is $11.68=n_{\mathrm{Si}}{ }^{2}$. The inset of Fig. 3(a) is cross sectional plot of $\sigma_{1}$ and $\varepsilon_{1}$ for a wave of $\omega=(2 \pi) 0.5 \mathrm{THz}$, shown by a dashed line in the $2 \mathrm{D}$ conductivity plot. 

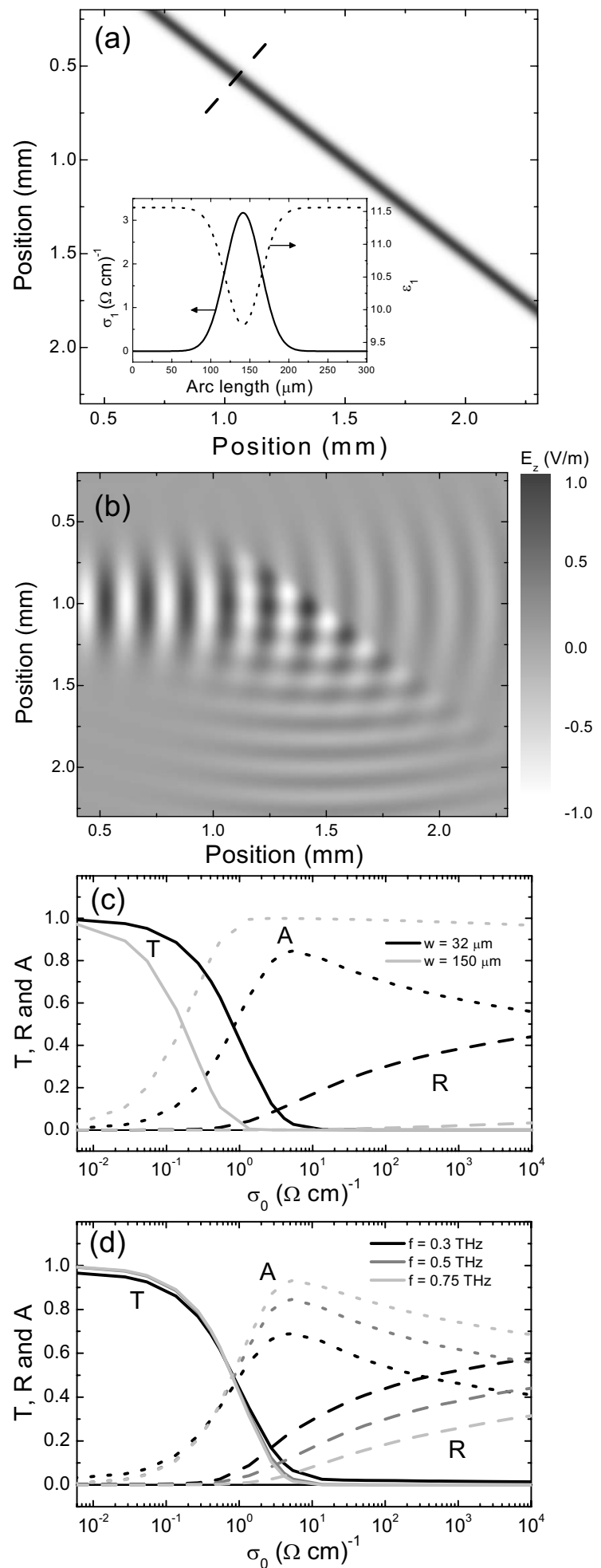

FIG. 3. Finite element 2D simulations using COMSOL of reflection and transmission through a Gaussian conductivity profile at $45^{\circ}$ to incoming wave. (a) The conductivity profile and a sliced view (dotted line) of $\sigma_{1}$ and $\varepsilon_{1}$ at $0.5 \mathrm{THz}$ (inset) for $w=32 \mu \mathrm{m}$. (b) $z$-component of the electric field incident on the conductivity profile in (a). The calculated power transmission (solid), reflection (dashed), and absorption (dotted) coefficients for (c) pump width, $w$, dependence at $0.5 \mathrm{THz}$ and (d) the frequency, $f$, dependence at $w=32 \mu \mathrm{m}$.

Figure 3(b) shows the out of plane component of the electric field, $E_{\mathrm{z}}$, calculated for $\sigma_{0}=3.25(\Omega \mathrm{cm})^{-1}$, corresponding to equal transmission and reflection of the incident field and verifies that a reflection is indeed possible by a 2D Gaussian conductivity profile in $\mathrm{Si}$. The extent of the trans- mission and reflection depends on $\sigma_{0}$ and $w$, as well as the frequency of the plane wave, $\omega$. Figure 3(c) plots the dependence of the power coefficients of transmission $(T)$, reflection $(R)$, and absorption (calculated as $A=1-T-R$ ) on peak conductivity, $\sigma_{0}$. We note that in our experiment we estimate $\sigma_{0}=5(\Omega \mathrm{cm})^{-1}$ from our pump fluence, corresponding to a $4 \%$ transmission and an $11 \%$ reflection of power according to the simulation in Fig. 3(c). These numbers serve only as a guide, however, since in the experiment the pump beam was of finite length and it was impossible to know the overlap between the $\mathrm{THz}$ beam and the pump beam. It is therefore likely that the transmitted pulse 1 sees a region of lower conductivity, and thus transmits more, as evidenced by $\Delta E$ for pulse 1 decreasing as $\Delta y$ increases in Fig. 2(a). Figure 3(d) shows the frequency dependence of the optical coefficients, showing decreasing reflection and increasing absorption for higher frequencies, as is expected.

In summary, we have demonstrated optically induced reflective THz quasioptics inside a PPWG and shown a beamsplitter as a simplified proof of principle. The technique relies on the creation of spatially well defined carrier profiles within the transparent waveguide that are sufficiently conducting to induce a reflection of the $\mathrm{THz}$ pulse. We have shown by finite element calculations that reflection coefficients greater than $30 \%$ can be achieved with conventional focusing optics well above the diffraction limit of the 1030 $\mathrm{nm}$ pump beam. These dynamic quasioptics can be switched on with a speed limited by the duration of the excitation pulse, and are reconfigurable on time scales determined by the lifetime of carriers in the semiconductor.

The authors would like to thank D. Turchinovich for helpful discussions and L. Ottaviano for the gold deposition. D. G. Cooke is grateful to DTU's H. C. Ørsted fellowship program and the authors acknowledge funding from TeraNova.

${ }^{1}$ M. Tonouchi, Nat. Photonics 1, 97 (2007).

${ }^{2}$ H.-T. Chen, W. J. Padilla, J. M. O. Zide, A. C. Gossard, A. J. Taylor, and R. D. Averitt, Nature (London) 444, 597 (2006).

${ }^{3}$ H.-T. Chen, J. F. O'Hara, A. K. Azad, A. J. Taylor, R. D. Averitt, D. B. Shrekenhamer, and W. J. Padilla, Nat. Photonics 2, 295 (2008).

${ }^{4}$ H. O. Moser, B. D. F. Casse, O. Wilhelmi, and B. T. Saw, Phys. Rev. Lett. 94, 063901 (2005).

${ }^{5}$ G. Gallot, S. P. Jamison, R. W. McGowan, and D. Grischkowsky, J. Opt. Soc. Am. B 17, 851 (2000).

${ }^{6} \mathrm{R}$. Mendis and D. Grischkowsky, Opt. Lett. 26, 846 (2001).

${ }^{7}$ K. L. Wang and D. M. Mittleman, Nature (London) 432, 376 (2004).

${ }^{8}$ G. de los Reyes, A. Quema, C. Ponseca, Jr., R. Pobre, R. Quiroga, S. Ono, H. Murakami, E. Estacio, N. Sarukura, K. Aosaki, Y. Sakane, and H. Sato, Appl. Phys. Lett. 89, 211119 (2006).

${ }^{9}$ A. L. Bingham and D. Grischkowsky, Appl. Phys. Lett. 90, 091105 (2007).

${ }^{10}$ W. Q. Zhu, A. Agrawal, and A. Nahata, Opt. Express 16, 6216 (2008)

${ }^{11}$ S. Coleman and D. Grischkowsky, Appl. Phys. Lett. 83, 3656 (2003).

${ }^{12}$ J. M. Dai, S. Coleman, and D. Grischkowsky, Appl. Phys. Lett. 85, 884 (2004).

${ }^{13}$ Z. P. Jian, J. Pearce, and D. M. Mittleman, Semicond. Sci. Technol. 20, S300 (2005)

${ }^{14}$ Y. Zhao and D. Grischkowsky, Opt. Lett. 31, 1534 (2006).

${ }^{15}$ A. L. Bingham and D. Grischkowsky, Opt. Lett. 33, 348 (2008).

${ }^{16}$ D. G. Cooke and P. Uhd Jepsen, Opt. Express 16, 15123 (2008).

${ }^{17}$ D. G. Cooke and P. Uhd Jepsen, Phys. Status Solidi A 206, 997 (2009).

${ }^{18}$ R. T. Hammond, J. Davis, and L. Bobb, J. Appl. Phys. 81, 1619 (1997).

${ }^{19}$ S. R. Keiding and D. Grischkowsky, Opt. Lett. 15, 48 (1990).

${ }^{20}$ D. Grischkowsky, S. Keiding, M. v. Exter, and C. Fattinger, J. Opt. Soc. Am. B 7, 2006 (1990). 